\title{
Finite Element Dynamic Bifurcation Buckling Analysis of Torispherical Head of BWR Contaiment Vessel Subjected to internal Pressure
}

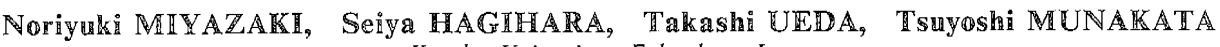 \\ Kyushu University, Fukuoka, Japan \\ Kunihisa SODA \\ Japan Atomic Energy Research Institute, Tokaimura, Japan
}

\begin{abstract}
$A B S T R A C T$
We calculate the bifurcation buckling pressure for the torispherical head of the Mark II type BWR containment vessel subjected to dynamically applied internal pressure, using a finite element program for a dynamic analysis. Three kinds of dynamic loadings, that is, step loading, ramp loading and pulse loading are considered in the present analys is. The minimum bifurcation buckling pressure is predicted for the respective loadings. The minimum bifurcation buckling pressure for dynamic loading is much lower than the bifurcation buckling pressure for static loading.
\end{abstract}

\section{INTRODUCTEON}

A torispherical shell is known to buckle with circumferential waves on a knuckle region, when it is subjected to excessive internal pressure (Bushnel1 1982; Galletly and Blachut 1985; Galletly 1986). The circumferential waves observed are caused by bifurcation buckling. A torispherical shell is utilized as a head of a BUR containment vessel. It may buckle due to overpressure caused by severe accidents. Dynamic loading may act on a containment vessel due to postulated hydrogen or steam explosion. Larger deflection is induced under dynamic loading than the deflection predicted by a static analysis (Stricklin, et al. 1971; Nagarajan and Popov 1975; Ball and Burt 1973). In general, the bifurcation load is lower in the case of dynamic loading than in the case of static loading. Therefore, it is important to obtain the bifurcation load for a torispherical head due to dynamic loading from the viewpoint of estimating safety margin of a BWR containment vessel under severe accident condition. In the present paper, we calculate the bifurcation buckling pressure for the torispherical head of the Mark II type BWR containment vessel, using a finite element program for a dynamic analysis. Three kinds of dynamic loadings, that is, step loading, ramp loading and pulse loading are considered in the present analysis. The minimum bifurcation buckling pressure is predicted for the respective loadings.

\section{METHOD OF ANALYSIS}

Elastic-plastic bifurcation buckling analysis under dynamic loading consists of two parts, that is, a pre-buckling dynamic deformation analysis and a bifurcation buckling analysis.

2.1 Pre-Buckling Dynamic Deformation Analysis

SMiRT 11 Transactions Vol. J (August 1991) Tokyo, Japan, (C) 1991 
In the pre-buckling dynamic deformation analysis of the axisyminetric mode, we used the finite element equation of motion. The geometric nonlinearity was taken into account in the strain-displacement relations, and the Ja flow theory with the isotropic strain hardening rule was used for the stressstrain relations in the plastic range (Miyazaki et al. 1987). Then, the finite element equation of motion of a total system is given as follows.

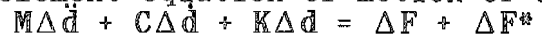

where, is a mass matrix, $\mathbb{C}$ is a damping matrix, $\mathbb{K}$ is a stiffness matrix, $\Delta P^{\prime}$ is an incremental nodal force vector, $\Delta$ Fin $^{3}$ is a residual force vector, $\Delta d$ is an incrememtal nodal displacement vector, $\Delta$ d is an incremental nodal velocity vector, and $\Delta$ dis an incremental nodal acceleration vector. The stiffuess matrix $K$ is composed of the followings.

$\mathrm{Ke}(0):$ elastic small displacement stiffness matrix,

$K_{p}(0):$ plastic stiffmess matrix,

$K(0)$ : initial displacement stiffness matrix,

$\mathbb{K}_{\sigma}(0)^{\text {: }}$ initial stress stiffness matrix.

where the superscript (0) indicates the axisymmetric mode.

The Newmark- $\beta$ method was applied to the time integration of equation (1). Then, the following equation is derived from the equation (1).

$$
\begin{aligned}
& \left(\frac{4}{\Delta \mathbb{t}^{2}} M+\frac{2}{\Delta t} \mathbb{C}+\mathbb{K}\right) \Delta \mathbb{N}=\Delta \mathrm{F}+\Delta \mathrm{F}
\end{aligned}
$$

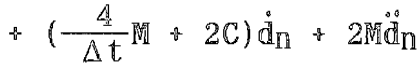

where, $\mathbb{C}$ was set equal to zero in the present analysis. We can obtain the incremental modal displacement vector at $n$ step by solving equation (2). The total nodal displacements are obtained by accumulating the incremental ones at each time step. The incremental nodal acceleration vector and the incremental nodal velocity vector can be calculated from the incremental nodal displacement vector.

\subsection{Deterningtion of Bifurcation Buckling}

To examine whether the bifurcation buckling with $n$ circurferential waves occurs or not, we stop time marching (Akkas 1972) and add the following infinitesimal displacement $\delta U$ to the equilibrium state obtained from the pre-buckling deformation analysis.

$$
\delta U=\{\delta u \delta w \delta v\}=\{\delta u(n) \cos \theta \quad \delta w(n) \cos \theta \quad \delta v(n) \operatorname{sinn} \theta\} \quad(3)
$$

where the infinitesimal displacement components $u$, w and $y$ denote the meridian in-plane, meridian out-of-plane, and circumferential displacements, respectively, and $\theta$ is the circumferential angle. The superscript (n) indicates a quantity relevant to the bifurcation buckling with circumferential waves. The bifurcation buckling with $n$ circumferential waves can be predicted when the following equation has a nontrivial solution.

$$
\mathrm{K}(\mathrm{n}) \delta \mathrm{U}=0
$$

where $K(n)$ is the stiffness matrix relevant to $n$ circumferential waves. This condition can be replaced by the following equation.

$$
\operatorname{det}[K(n)]=0
$$

where det [ ] is the determinant of a matrix. It is assumed that loading condition continues even after the infinitesimal displacement $\delta \mathrm{U}$ is given to the system, which is so-called consistent loading (Bushnell 1974). In obtaining $K(n)$, we need the stress-strain relations in the plastic range. The incremental form of the $\mathrm{J}_{2}$ deformation theory (Miyazaki et al. 1989) was adopted as the plastic theory in the present bifurcation buckling analysis, because it gives more appropriate results than the $\mathrm{J}_{2}$ flow theory, as pointed out by Bushnell. (Bushnell 1974), and Roche and Autrusson (Roche and Autrusson 1986).

\section{RESULTS AND DISCUSSION}

Figure 1 shows the dimensions of the torispherical shell of the head employed in the Mark II type BWR containment vessel (Greimann et al. 1982). The dimensions of analyzed one are as follows; crown radius of the shell, 
$R_{S}=8.734 \mathrm{~m}$, knuckle radius of the shell, $r=1.657 \mathrm{~m}$, diameter of the attached cylindrical shell, $D=9.652 \pi$, height of the attached cylindrical shell, $\mathrm{L}=2.244 \mathrm{~m}$ and thickness of the shell, $\mathrm{t}=0.024 \mathrm{~m}$.

A bilinear approximation of the stress-strain curves was used in the present analysis. The Young's modulus $\mathrm{E}=1.90 \times 105 \mathrm{MPa}$, the Poisson's ratio $\nu=0.3$, the yield stress $\sigma \mathrm{y}=3.98 \times 10^{2} \mathrm{MPa}$, the rate of strain hardening $\mathrm{H}^{\circ}=1.50 \times 10^{3} \mathrm{MPa}$ and the density $\rho=7800 \mathrm{~kg} / \mathrm{m} 3$ were determined from the data of Isozaki et al. (Isozaki et al. 1987).

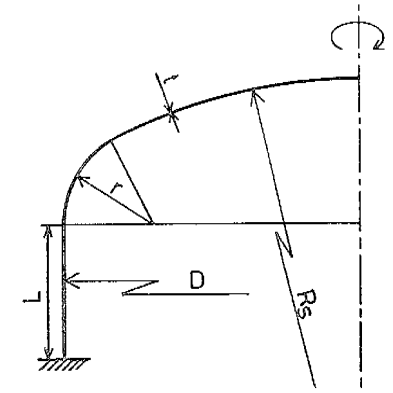

Fig.1 Dimensions of the torispherical shell

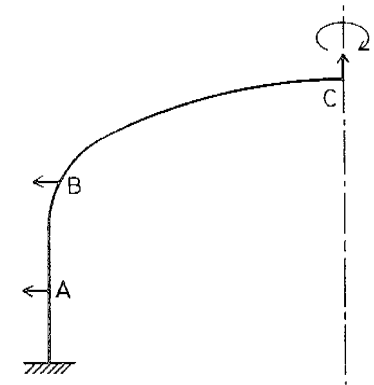

Fig. 2 The locations and the directions of displacements plotted in following figures

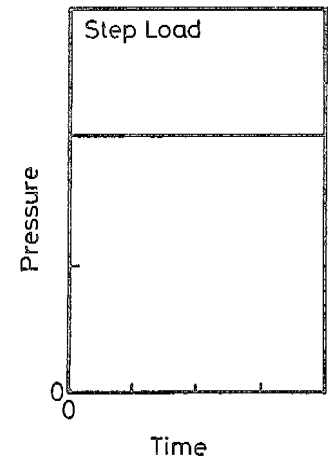

(a)

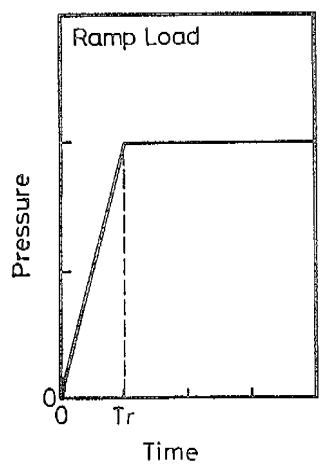

(b)

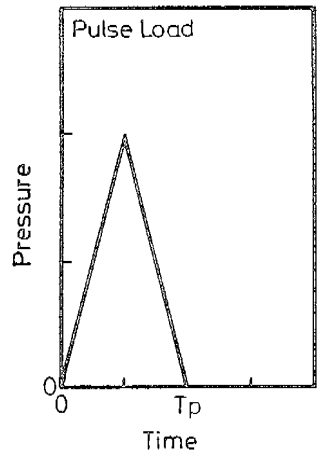

(c)

Fig. 3 Three kinds of dynamic loadings

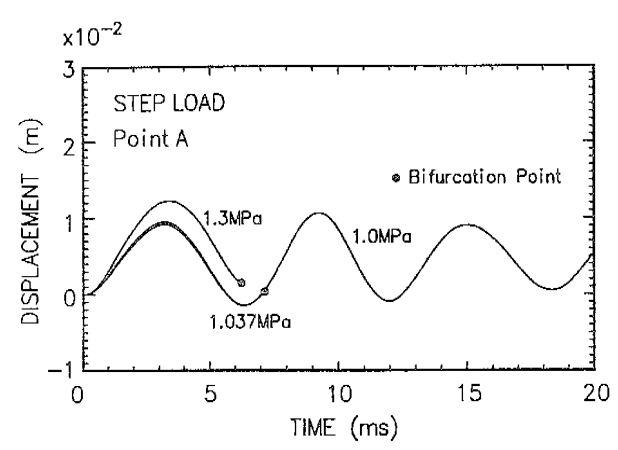

Fig.4 The radial displacement for the point $A$ versus time curves

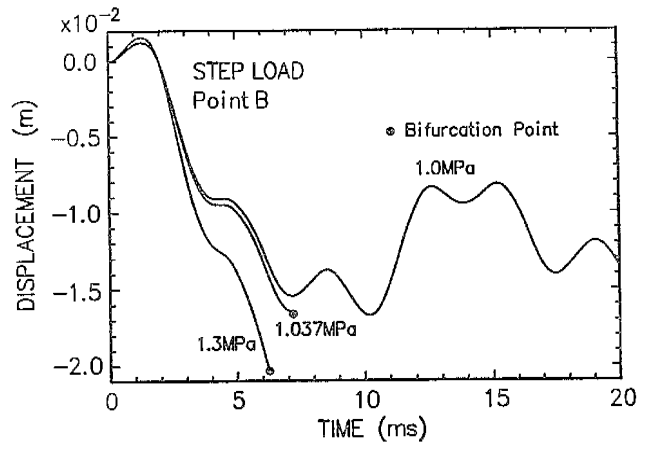

Fig.5 The radial displacement for the point $B$ versus time curves 
A half of the shell was divided into 180 axisymetric thin shell elements. A fine finite element mesh was used at the knuckle region.

Three points were selected to show the results of analysis, as shown in Figure 2, where the points $A, B$ and $C$ are the middie of the attached cylindrical shell, the middle of the knuckle region and the top of the torispherical shell, respectively. The arrows in Figure 2 indicate the positive direction of the displacement at the respective locations. Three kinds of dynamic loadings, that is, step loading, ramp loading and pulse loading were considered in the present analysis as the internal pressure applied to the torispherical shell. The schematic figures of these loadings are given in Figure 3 where $T_{r}$ and $T p$ denote the ramp time and the pulse width, respectively. The displacement versus time curves for the points $A, B$ and $C$ are respectively shown in Figures 4,5 and 6 in the case of step loading. As shown in these figures, the minimum bifurcation buckling pressure is $1.037 \mathrm{MPa}$ for step loading, and the bifurcation buckling can not be induced by 1.0 MPa interna1 pressure. It seems that the displacements at the point $\mathrm{A}$, which is the middle of the attached cylindrical shell, oscillates regularly, while those at the other points oscillate irregularly. The period of the regular oscillation is in good agreement with the natural period of the attached cylindrical shell calculated by the simplified method, $6.61 \mathrm{msec}$ (Isozaki, $\mathbb{T}$. et $2 \mathbb{1}$. 1988). The bifurcation buckling seerns to be induced by the large negative displacement of the knuckle region, as shown in Figure 5. We confirmed large plastic zone extended in the knuckle region at the bifurcation buckling point in each case.

Figure 7 shows the circumferemtial membrane force at the knuckle region vs. time curves. The bifurcation buckling occurs when the circumferential membrane force at the knuckle region is negative.

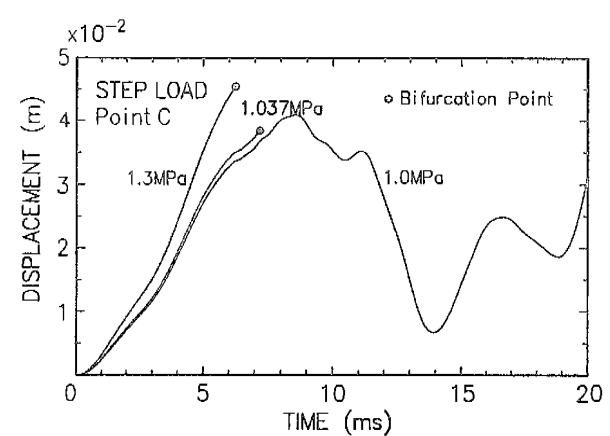

Fig.6 The axial displacement for the point $\mathrm{C}$ versus time curves

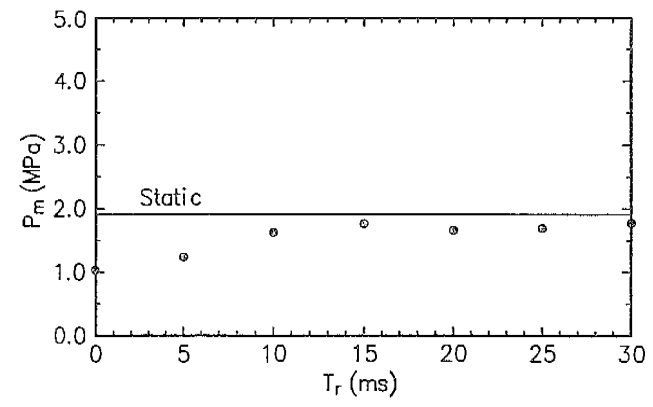

Fig. 8 The minimum bifurcation buckling pressure in the case of ramp loading

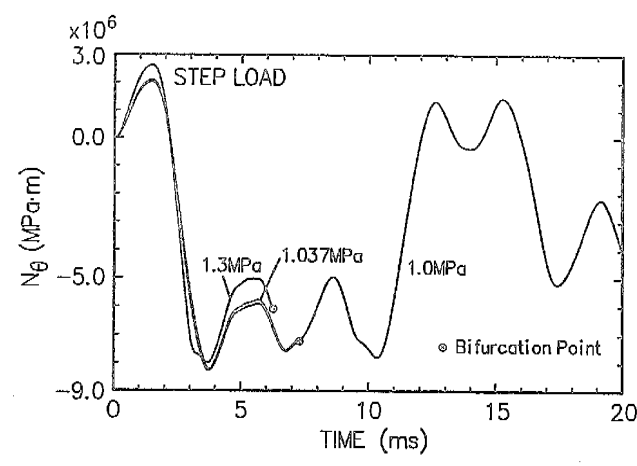

Fig.7 The circumferential membrane force for the point $\mathbb{B}$ versus time curves

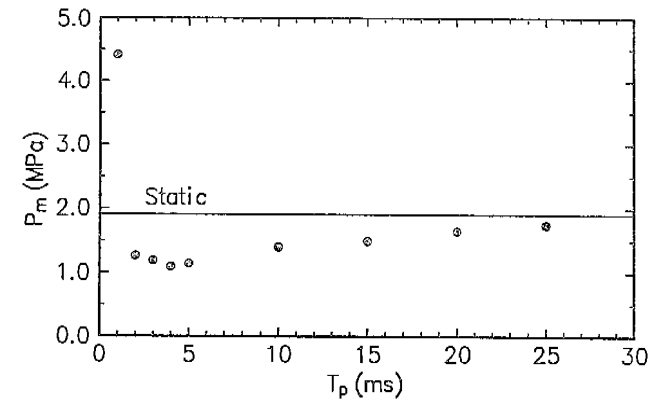

Fig.9 The minimum bifurcation buckling pressure in the case of pulse loading 


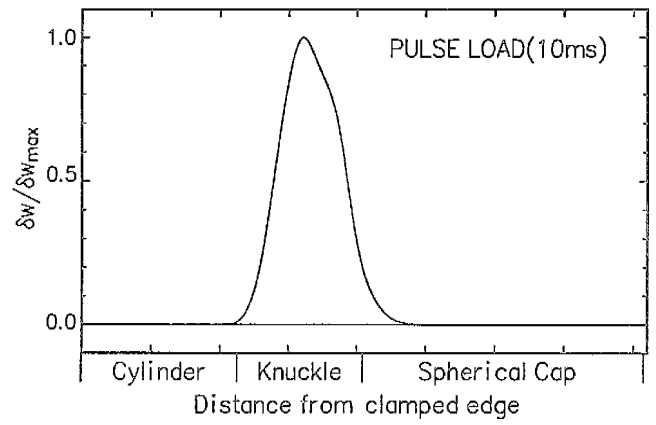

Fig.10 The radial amplitude of bifurcation buckling mode in the case of pulse

loading with $\mathrm{T}_{\mathrm{p}}=10 \mathrm{msec}$

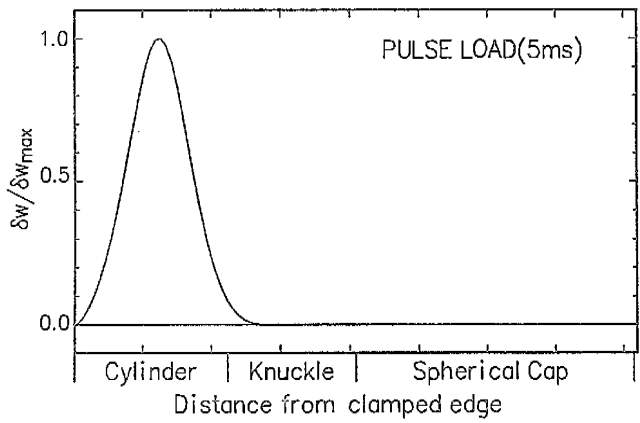

Fig. 11 The radial ariplitude of bifurcation buckling mode in the case of pulse loading with $\mathrm{T}_{\mathrm{p}}=5$ insec

The minimum bifurcation buckling pressure versus the ramp time $\mathrm{Tr}$ is shown in Figure 8 for ramp loading. The bifurcation buckling pressure and the circumferential maves obtained from a static bifurcation buckling analysis are $1.91 \mathrm{MPa}$ and $n=22-24$, respectively. As shown in Figure 8 , the minimum bifurcation buckling pressures for the dynamic loading are lower than the bifurcation buckling pressure for static loading. When the ramp time becomes longer, the minimum bifurcation buckling pressure for ramp loading approaches to the static bifurcation buckling pressure. On the other hand, it approaches to the minimum bifurcation buckling pressure for step loading, Tr $=0$, when the ramp time decreases to zero. The minimum bifurcation buckling pressure versus the pulse width, Tp, for pulse loading is shown in Figure 9. It is found from the figure that the minimum bifurcation buckling pressure for pulse loading gradually increases with the increase of the pulse width after it has the minimum point around $T_{p}=4$ msec and approaches to the static bifurcation buckling pressure. On other hand, the minimum bifurcation buckling pressure tends to infinite when the pulse width approaches to zero.

Figure 10 and 11 show the bifurcation buckling mode for the pulse loading with $\mathrm{T} p=10 \mathrm{msec}$ and for the pulse loading with $\mathbb{T} p=5$ msec, respectively. It is found that the circumferential waves are observed at the knuckle region for the pulse loading with $\mathrm{Tp}=10$ msec, but they are observed at the middle of the attached cylindrical shell for the pulse loading with $\mathrm{T}_{\mathrm{p}}=5 \mathrm{msec}$. It is supposed that in the latter case the bifurcation buckling is due to the large deformation to the inward radial direction of the attached cylindrical shell caused by resonance because the pulse width $\mathrm{Tp}=5$ msec is nearly corresponding to the natural period of the attached cylindrical shell.

\section{CONCLUDHNG REMARKS}

We calculated the bifurcation buckling pressure for the torispherical head of the Mark II type BWR containment vessel, using a finite element program for a dynamic analysis. Three kinds of dynamic loadings, that is, step loading, ramp loading and pulse loading are considered as applied internal pressure in the present analysis. The minimum bifurcation buckling pressure for dynamic $\mathbb{l}$ oading is much lower than the bifurcation buckling pressure for static loading. Especially, the minimum bifurcation buckling pressure for step loading is about a half of the bifurcation buckling pressure for static loading. When the ramp time or the pulse width becomes longer, the minimum bifurcation buckling pressures for dynamic loadings approach to the static bifurcation buckling pressure. In the case of pulse loading, the minimum bifurcation buckling pressure tends to infinite when the pulse width approaches to zero. 


\section{REFERENCES}

Akkas, N, 1972. Asymmetric Buckling Behavior of Spherical Caps under Uniform Step Pressure. ASME Journal of Applied Mechanics.39: pp.293-294.

Ball,R.E. and Burt,J.A. 1973. Dynamic Buckling of Shallow Spherical Shells. ASME Journal of Applied Mechanics. 40: pp.411-416.

Bushnell, D。 1974. Bifurcation Buckling of Shells of Revolution Including Large Deflections, Plasticity and Creep. International Journal of Solids and Structures. 10: pp.1287-1305.

Bushne11,D. 1982. Plastic Buckling of Various Shells. ASME Journal of Pressure Vessel Technology 104: pp.51-72.

Galletly,G.D. and Blachut.J. 1985. Torispherical Shells under Internal Pressure - Failure Due to Asymetric Plastic Buckling or Axisymmetric Yielding. Proceedings of the Institution of Mechanical. Engineers. 199-C3: pp. $225-238$.

Galletly,G.D. 1986. A Simple Design Equation for Preventing Buckling in Fabricated Torispherical Shells under Internal Pressure. ASWE Journal of Pressure Vessel Technology. 108: pp.521-526.

Greimann, L, G, et al, 1982. Reliability Analysis of Steel-Containment Strength. NUREG/CR-2442。

Isozaki, T. et al. 1987. Structural Analysis of a Japanese BWR MARK-I Containment under Internal Pressure Loading, Nuclear Engineering and Design. 104: pp.365-370.

Isozaki, T, et al. 1988. Structural Analysis of a Japanese PWR Steel Containment under Inner Pressure Loading. Proceedings of the Forth Workshop on Containment Integrity. June 15-17, (1988), Washington, D.C.

Miyazaki, N, et al, 1987. Elastic-Plastic Creep Buckling Analysis of a Cylindrical Shell under Axial Compression by the Finite Element Method. Technology Reports of the Kyushu University 60-2: pp.193-200

Miyazaki,N. et al. 1989. Elastic-Plastic Creep Buckling Analysis of Partial Spherical Shell by the Finite Element Method. Computational Plasticity Proceedings of the Second International Conference. September 18-22: pp.687-698.

Nagarajan,S. and Popov, E.P. 1975. Non-Linear Dynamic Analysis of Ax isymetric Shells. International Journal for Numerical Methods in Engineering. 9: pp.535-550.

Roche,R.L. and Autrusson,B. 1986. Experimental Tests on Buckling of Torispherical Heads and Methods of Plastic Bifurcation Analysis. ASME Journal of Pressure Vessel Technology. 108: pp.138-145.

Stricklin,J.E.,et al. 1971. Nonlinear Dynamic Analysis of Shells of Revolution by Matrix Displacement Method. AlAA Journal 9-4: pp.629-636. 